\title{
THE HOMOLOGY OF SYMMETRIC PRODUCTS
}

BY

\section{R. JAMES MILGRAM}

In this paper we compute the homology groups for the various symmetric products of any space $X$ of finite type. Thus we complete the calculations begun by M. Morse, Smith and Richardson in the 1930's and carried dramatically forward by N. Nakaoka in a series of papers dating from 1955. Our methods are essentially geometric in nature and are based on a close examination of the geometry of the topological bar construction introduced in [10]. Indeed it was the study of the symmetric products which led to [10], but the exposition given here is selfcontained.

(1) The $m$-fold symmetric product $S P^{m}(X)$ is the set of all unordered $m$-tuples $\left\langle x_{1}, \ldots, x_{m}\right\rangle$ of points in $X$. Equivalently, $S P^{m}(X)$ is the orbit space of the Cartesian product $X^{m}$ under the action of $\mathscr{S}_{m}$, the symmetric group on $m$ letters. It has the quotient topology.

Let a base point $* \in X$ be given, then there is an inclusion

$$
j: S P^{m}(X) \subset S P^{m+1}(X)
$$

given on points by

$$
j\left(\left\langle x_{1}, \ldots, x_{m}\right\rangle\right)=\left\langle *, x_{1}, \ldots, x_{m}\right\rangle .
$$

Moreover, there is the evident associative and abelian pairing

$$
M: S P^{m}(X) \times S P^{n}(X) \rightarrow S P^{n+m}(X)
$$

defined on points by

$$
M\left(\left\langle x_{1}, \ldots, x_{m}\right\rangle,\left\langle x_{m+1}, \ldots, x_{m+n}\right\rangle\right)=\left\langle x_{1}, \ldots, x_{m}, x_{m+1}, \ldots, x_{m+n}\right\rangle .
$$

$M$ respects inclusion in the sense that we have the commutative diagram

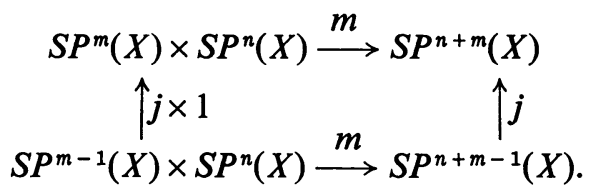

In particular $M$ induces a Pontrjagin multiplication in the set

$$
\mathscr{R}(X, \Gamma)=\sum_{m=1}^{\infty} H_{*}\left(S P^{m}(X), S P^{m-1}(X) ; \Gamma\right)
$$

Received by the editors April 26, 1966 and, in revised form, November 22, 1967. 
where $\Gamma$ is a suitable coefficient ring (for example $Z$, or $Z_{p}$ ) and a result of Steenrod [3] for connected $X$

$$
H_{*}\left(S P^{n}(X) ; \Gamma\right) \cong \sum_{m=1}^{n} H_{*}\left(S P^{m}(X), S P^{m-1}(X) ; \Gamma\right)
$$

shows that it is, in fact, sufficient for our purposes to calculate $\mathscr{R}(X, \Gamma)$.

(2) $\mathscr{R}(X, \Gamma)$ is a bigraded ring, that is, if we set

$$
\mathscr{R}_{i, m}=H_{i}\left(S P^{m}(X), S P^{m-1}(X) ; \Gamma\right)
$$

then

$$
M_{*}\left(\mathscr{R}_{i, m} \otimes \mathscr{R}_{s, t}\right) \subset \mathscr{R}_{i+s, m+t} .
$$

Moreover, since $M$ is commutative and associative so is $M_{*}$.

In case $X$ is a Moore space $A(\Pi, n)(n \geqq 1)$ it follows from the Thom-Dold theorem [8] that $S P^{\infty}(A(\Pi, n))=\lim \operatorname{dir} S P^{n}(A(\Pi, n))$ is an Eilenberg-MacLane space $K(\Pi, n)$. Thus $\mathscr{R}(A(\Pi, n), \Gamma)$ is just a bigraded version of the Pontrjagin ring of $K(\Pi, n)$ which has been completely determined by H. Cartan [2], [3]. Thus the problem reduces to finding a proper bigrading for Cartan's results. In fact it turns out, with $\Pi=\Gamma=Z_{p}$ where $p$ is prime, that generators (in homology) are in 1-1 correspondence with the admissible monomials $\mathscr{P I}^{\mathrm{I}}$ in the Steenrod algebra $\mathscr{A}(p)$ of excess $<n$, and the bidegree of the generator is $\left(\left|\mathscr{P}^{I}\right|+n, l\right)$. Here $\left|\mathscr{P}^{I}\right|$ is the degree of $\mathscr{P}^{I}$ and $l$ is the "length" of $\mathscr{P}^{I}$. Thus if

$$
\mathscr{P}^{I}=\beta^{\varepsilon_{1}} \mathscr{P}^{i_{1}} \beta^{\varepsilon_{2}} \mathscr{P} i_{2} \ldots \mathscr{P} i_{1} \beta^{\varepsilon_{j+1}}
$$

then $l=p^{j}$, and we have

THEOREM 5.2. $\mathscr{R}(A(\Pi, n))$ is ring isomorphic to $H_{*}(G(\Pi, n))$ where $G(\Pi, n)$ is a certain (explicitly given) tensor product of bigraded differential algebras of four kinds (divided polynomial algebra $P(m, r)$, special divided polynomial algebras $P_{h}(m, r)$, Grassmann algebras $E(m, r)$, and special Grassmann algebras $\left.E_{h}(m, r)\right)$.

Of course these are just the bigraded versions of the algebras introduced by Cartan, and the double indices specify the bidegree of the generators.

This is our main result. The special case when $\Pi$ is cyclic is proved in $\S 4$. The proof is then completed in $\$ 5$ where we also use a result of Dold [4] to compute $H_{*}\left(S P^{m}(X)\right)$ for arbitrary $X$ of finite type. Except for the proof of an algebraic result, the Cartan-Moore theorem (Theorem 1.1) the paper is entirely self-contained, in fact together with the results of [10] our techniques give the most direct path to the cohomology of the Eilenberg-MacLane spaces known to the author $\left({ }^{1}\right)$.

( ${ }^{1}$ A short proof of the last part of the Cartan-Moore theorem can be given using the Eilenberg-Moore spectral sequence (filtering by bar degree). Indeed if $f: A \rightarrow B$ is a map of D.G.A. algebras so $f_{*}: H(A) \rightarrow H(B)$ is an isomorphism then $E^{1}(f): \mathscr{E}^{1}(B(A)) \rightarrow \mathscr{E}^{1}(B(B))$ is already an isomorphism. Finally, it is easy to show that two different resolutions of the same D.G.A. algebra have isomorphic homology. 
(3) To illustrate our results we now use them to calculate the two primary cohomology of $\mathscr{S}_{4}$ with untwisted coefficients. Since the singular locus of $\left(S^{2 n}\right)^{4}$ under $\mathscr{S}_{4}$ has dimension $6 n$ it follows that

$$
H^{i}\left(\mathscr{S}_{4} ; \Gamma\right) \cong H_{8 n-i}\left(S P^{4}\left(S^{2 n}\right) ; \Gamma\right)
$$

for $i<2 n-1$. On the other hand, with twisted $Z$ or $Z_{p}$ coefficients it is easy to see that

$$
H^{i}\left(\mathscr{S}_{4} ; \Gamma\right) \cong H_{8 n+4-i}\left(S P^{4}\left(S^{2 n+1}\right) ; \Gamma\right)
$$

in the same range of dimension.

Hence, since $H_{j}\left(S P^{4}\left(S^{2 n}\right), S P^{3}\left(S^{2 n}\right)\right) \cong H_{j}\left(S P^{4}\left(S^{2 n}\right)\right)$ for $j>6 n+1$ it follows that we can use $\mathscr{R}\left(S^{2 n} ; \Gamma\right)$. In particular we specify all generators of $\mathscr{R}\left(S^{2 n} ; Z_{2}\right)$ having second degree $\leqq 4$. These correspond to $i, S q^{j}(i) \quad 1<j \leqq 2 n$, and $S q^{2 r+s} S q^{r}(i)$ with $r>1$ and $r+s \leqq 2 n$. Moreover each of these generates an exterior algebra, hence all elements of bidegree 4 in $\mathscr{R}\left(S^{2 n}, Z_{2}\right)$ are contained in

$$
E(e) \otimes E\left(f_{2}\right) \otimes \cdots \otimes E\left(f_{2 n}\right) \otimes E\left(g_{2,0}\right) \otimes \cdots \otimes E\left(g_{i, j}\right) \otimes \cdots,
$$

where $e$ is dual to $i$ (bidegree $(2 n, 1)) f_{j}$ is dual to $S q^{j}$ (bidegree $(2 n+j, 2)$ ) and $g_{i, j}$ is dual to $S q^{2 i+j} S q^{i}$ (bidegree $(2 n+3 i+j, 4)$ ).

Thus a $Z_{2}$ basis for all elements with second degree 4 are the $f_{i} \otimes f_{j} i<j$, and the $g_{i, j}$. We can write

$$
\begin{aligned}
\operatorname{dim} f_{i} \otimes f_{j} & =8 n-(2 n-i)-(2 n-j), \\
\operatorname{dim}\left(g_{i, j}\right) & =8 n-3\{2 n-(i+j)\}-2 j,
\end{aligned}
$$

and setting $a=2 n-i, b=2 n-j, c=2 n-(i+j)$ and passing to the limit over $n$ we see that an additive basis for $H^{*}\left(\mathscr{S}_{4}, Z_{2}\right)$ is

$$
\tilde{f}_{a} \tilde{f}_{b}, \quad \text { with } b>a \text { and } \tilde{g}_{c, j}
$$

where $\operatorname{dim}\left(\tilde{f}_{a} \tilde{f}_{b}\right)=a+b, \operatorname{dim}\left(\tilde{g}_{c, j}\right)=3 c+2 j$.

(4) We now study the ring structure in $H^{*}\left(\mathscr{S}_{4}, Z_{2}\right)$.

Remark. Consider the "2-fold suspension"

$$
s_{2}: \Sigma^{2}\left(S P^{r}(X)\right) \rightarrow S P^{r}\left(\Sigma^{2} X\right)
$$

defined in the evident way (see $\S 3$ ). When $X$ is $S^{2 n}$ it follows from 3.4 that $\left(s_{2}\right)_{*}$ induces a map of degree $+3, \mu: H^{*}\left(\mathscr{S}_{4}\right) \rightarrow H^{*}\left(\mathscr{S}_{4}\right)$, and it is not hard to show (from the proof of 3.4 and the fact that $\left(s_{2}\right)_{*}$ is obtained from a cell map) that $\mu(a)=a \cup w$ where $w=\mu(1)$.

On the other hand the map

$$
M: S P^{2}\left(S^{2 n}\right) \times S P^{2}\left(S^{2 n}\right) \rightarrow S P^{4}\left(S^{2 n}\right)
$$

gives rise to a map

$$
m: H^{*}\left(\mathscr{S}_{2}\right) \otimes H^{*}\left(\mathscr{S}_{2}\right) \rightarrow H^{*}\left(\mathscr{S}_{4}\right)
$$


and it is easy to verify that $m$ is just the transfer homomorphism induced by the inclusion

$$
I: \mathscr{S}_{2} \oplus \mathscr{S}_{2} \rightarrow \mathscr{S}_{4} .
$$

From these remarks and some further study of $\tilde{g}_{0,1}$ we can now show that $H^{*}\left(\mathscr{S}_{4}, Z_{2}\right)$ is ring isomorphic to the polynomial algebra in 3 variables

$$
P(w, g, f)
$$

modulo the single relation $w f=0\left(w=\mu(1)\right.$ corresponds to $\tilde{g}_{1,0}, g$ corresponds to $\tilde{g}_{0,1}$ and $f$ is $\tilde{f}_{0} \cdot f_{1}$ ).

(5) To find the order of the various cohomology classes above we use the information implicit in the chain complexes given in 2.1 to 2.4 on the Bockstein cohomology operation $\beta_{2}$ corresponding to the exact sequence

$$
0 \rightarrow Z_{2} \rightarrow Z_{4} \rightarrow Z_{2} \rightarrow 0 .
$$

We find $\beta_{2}(f)=f^{2}, \beta_{2}(g)=w+f g$. Thus the homology of $H^{*}\left(\mathscr{S}_{4}, Z_{2}\right)$ with respect to $\beta_{2}$ is

$$
P\left(g^{2}\right) \otimes E(f g) .
$$

Thus all other elements have order exactly 2 . Moreover $\beta_{4}(f g)=g^{2}$, and we can now read off the 2-primary cohomology of $\mathscr{S}_{4}$ with integer coefficients.

We defer further details and more extensive calculations of $H^{*}\left(\mathscr{S}_{n}\right)$ for all $n$ to a further paper.

I would like to take this opportunity to thank Professors E. Calabi and A. Aeppli for many helpful conversations-indeed this article is largely the content of my thesis written under Calabi's direction. I would also like to thank A. Mayer for renewing my interest in this problem.

1. Cartan's theory of constructions. Let $A$ be an associative algebra with unit over the ring $\Lambda\left(Z\right.$ or $\left.Z_{p}\right)$. It is bigraded in case $A$ is a direct sum

$$
A=\sum_{i=0}^{\infty} \sum_{j=0}^{\infty} A_{i j}
$$

and

$$
A_{i j} \cdot A_{k l} \subset A_{i+k, j+l} .
$$

There is a natural grading associated to the bigrading,

$$
A_{\jmath}=\sum_{k} A_{j k}
$$

and $A$ is commutative in case

$$
a \cdot b=(-1)^{p q} b \cdot a
$$

if $a \in A_{p}, b \in A_{q}$. 
$\Lambda$ is regarded as a bigraded ring when we set $\Lambda_{00}=\Lambda, \Lambda_{i j}=0$ if $i \neq j \neq 0$. The fact that $A$ has a unit implies there is a map $\eta: \Lambda \rightarrow A$, and we assume it is a map of bigraded rings. $A$ will be called augmented if there is a map $\varepsilon: A \rightarrow \Lambda$ of bigraded rings so that $\varepsilon \eta=\mathrm{id}$.

If $A$ is an augmented, commutative, bigraded algebra over $\Lambda$ it will be called a D.B.A-algebra in case there is a derivation $\partial$ in $A$ of degree $(-1,0)$, i.e.

$$
\begin{gathered}
\partial: A_{i j} \rightarrow A_{i-1, j}, \\
\partial(a \cdot b)=(\partial a) b+(-1)^{p} a \cdot \partial b \quad \text { if } a \in A_{p}
\end{gathered}
$$

and, if $\Lambda$ is regarded as a bigraded algebra with trivial derivation, $\varepsilon$ is a chain map.

Note that the tensor product of two D.B.A-algebras over $\Lambda$ is again a D.B.Aalgebra if we define a bigrading by

$$
\left(A \otimes_{\Lambda} B\right)_{i j}=\sum_{k+r=i ; s+t=j} A_{k s} \otimes B_{r t}
$$

and an augmentation by $\varepsilon(a \otimes b)=\varepsilon(a) \varepsilon(b)$.

A construction is a triple of D.B.A.-algebras $(A, N, M)$ with $M=A \otimes_{\Lambda} N$ as a bigraded augmented ring (however, not necessarily as a chain complex) such that:

(1) The injection $A \rightarrow A \otimes 1$ is a map of D.B.A-algebras.

(2) The projection $\pi: M \rightarrow N$ defined by $\pi(a \otimes b)=\varepsilon(a) b$ is a D.B.A-map.

(3) $\varepsilon_{*}: H_{*}(M) \rightarrow \Lambda$ is an isomorphism, that is, $M$ is acyclic over $\Lambda$.

$A$ will be called the initial algebra and $N$ the final algebra of the construction, $\bar{N}$ is $\operatorname{ker}(\varepsilon) \cap N$.

A special construction is a construction with a $\Lambda$-homomorphism $s$ (a contracting homotopy) of bidegree $(1,0)$ (not necessarily a ring homomorphism) which satisfies

(1) $s^{2}=0$,

(2) $s \partial+\partial s=1-\eta \varepsilon$,

(3) $1 \otimes \bar{N} \subset s(M), s(M) \cdot s(M) \subset s(M)$.

THEOREM 1.1 (CARTAN, MoORE). Let $(A, N, M)$ be a construction and $\left(A^{\prime}, N^{\prime}, M^{\prime}, s\right)$ a special construction. Suppose there is a D.B.A-map $g: A \rightarrow A^{\prime}$, then it may be extended to a unique D.B.A-map $\tilde{g}: M \rightarrow M^{\prime}$ so that $\tilde{g}(1 \otimes N) \subset s\left(M^{\prime}\right)$; hence to a unique homomorphism $\bar{g}: N \rightarrow N^{\prime}$. Moreover if $H_{0}(A)=\Lambda$ and $g_{*}: H_{*}(A) \rightarrow H_{*}\left(A^{\prime}\right)$ is an isomorphism the same is true of $\bar{g}_{*}$.

[The proof does not have to be changed in any essential way from that in [3] for D.G.A-algebras, and is thus omitted.]

THEOREM 1.2 (CARTAN). Let $(A, N, M),\left(A^{\prime}, N^{\prime}, M^{\prime}\right)$ be constructions, then there exists a construction

$$
\left(A \otimes_{\Lambda} A^{\prime}, N \otimes_{\Lambda} N^{\prime}, M^{\prime \prime}\right)
$$


Proof. Let $M^{\prime \prime}=\left(A \otimes A^{\prime}\right) \otimes\left(N \otimes N^{\prime}\right)$ with $\partial$ defined using the "shuffle" isomorphism

$$
M \otimes M^{\prime} \rightarrow M^{\prime \prime}
$$

Given a D.B.A-algebra $A$, let $\bar{A}=\operatorname{ker} \varepsilon$, and define $B(A)=\Lambda+\bar{A}+\bar{A} \otimes_{\Lambda} \bar{A}$ $+\cdots+\bar{A} \otimes_{\Lambda} \cdots \otimes_{\Lambda} \bar{A}+\cdots$. A new bigrading is defined in $B(A)$ as follows: if $a_{1} \otimes \cdots \otimes a_{n}$ has bidegree $(i, j)$ in the $\otimes$ bigrading its bidegree in $B(A)$ is $(i+n, j)$. An augmentation in $B(A)$ is defined by $\varepsilon \mid \Lambda$ is the identity, and $\varepsilon \mid B(A)_{i j}$ is the 0 -map for $i$ or $j$ greater than 0 .

Let $\alpha$ be an element of the symmetric group $\mathscr{S}_{m}$. For each such $\alpha$ there is a chain map shuff $(\alpha) \bar{A} \otimes \cdots \otimes \bar{A}$ to itself which permutes factors (with the appropriate signs). Using these maps we define a commutative, associative multiplication in $B(A)$ by

$$
\begin{aligned}
{\left[a_{1} \otimes \cdots \otimes a_{n}\right] \cdot\left[a_{n+1} \otimes\right.} & \left.\cdots \otimes a_{n+m}\right] \\
& =(-1)^{m \sum_{j=1}^{n} \operatorname{dim}\left(a_{j}\right)} \sum_{\alpha \in S(n, m)}(\operatorname{sgn} \alpha)\left[\operatorname{shuff} \alpha\left(a_{1} \otimes \cdots \otimes a_{n+m}\right)\right]
\end{aligned}
$$

where $S(n, m)$ is the set of all $(n, m)$ shuffles.

We now define a derivation in $A \otimes_{\Lambda} B(A)$ by

$$
\begin{aligned}
\partial\left(a \otimes\left[a_{1} \otimes \cdots \otimes a_{n}\right]\right)=\partial a \otimes\left[a_{1} \otimes \cdots \otimes a_{n}\right] \\
+(-1)^{\operatorname{dim} a+\operatorname{dim} a_{1}(n-1)} a a_{1} \otimes\left[a_{2} \otimes \cdots \otimes a_{n}\right] \\
+(-1)^{\operatorname{dim} a} a \otimes\left\{\sum_{j=1}^{n-1}(-1)^{j}\left[a_{1} \otimes \cdots \otimes a_{j} a_{j+1} \otimes \cdots \otimes a_{n}\right]\right. \\
\left.\quad+\sum_{j=1}^{n-1}(-1)^{n+S(j)}\left[a_{1} \otimes \cdots \otimes \partial a_{j} \otimes \cdots \otimes a_{n}\right]\right\}
\end{aligned}
$$

where $S(j)=\sum_{k<j} \operatorname{dim} a_{k}$.

A contracting homotopy $s$ is defined by

$$
s\left(a \otimes\left[a_{1} \otimes \cdots \otimes a_{n}\right]\right)=(-1)^{n \operatorname{dim} a_{1}} \otimes\left[\tilde{a} \otimes a_{1} \otimes \cdots \otimes a_{n}\right]
$$

where $\tilde{a}=a-n \varepsilon(a)$. It is easy to check that

$$
s \partial+\partial s=1-\eta \varepsilon .
$$

Moreover, $\partial$ is a derivation with respect to the multiplication in $A \otimes B(A)$. Thus by projection $\partial$ induces a boundary operator $\partial_{-}$in $B(A)$ which is also a derivation. We have

$$
\begin{aligned}
\partial_{-}\left[a_{1} \otimes \cdots \otimes a_{n}\right]= & \sum_{k=1}^{n-1}(-1)^{k}\left[a_{1} \otimes \cdots \otimes a_{k} a_{k+1} \otimes \cdots \otimes a_{n}\right] \\
& +\sum_{k=1}^{n}(-1)^{n+S(k)}\left[a_{1} \otimes \cdots \otimes \partial a_{k} \otimes \cdots \otimes a_{n}\right] .
\end{aligned}
$$

Thus, the triple $(A, B(A), A \otimes B(A), s)$ becomes a special construction. It is known as the bar construction. 
REMARK. Our boundary operators differ in sign from those usually used in the definition of the bar construction, as does the contracting homotopy $s$, so as to make clearer the geometric interpretations which arise in $\$ 3$.

\section{Constructions for special D.B.A-algebras.}

Definition 2.1. The Grassmann algebra $E(n, m)$ is the D.B.A-algebra with a single generator e of bidegree $(n, m),(e \cdot e=0)$ and trivial derivation.

Definition 2.2. The divided polynomial algebra $P(2 n, m)$ is the D.B.A-algebra with generators $p_{1}, p_{2}, \ldots, p_{n}, \ldots, p_{i}$ of bidegree $(2 n i, i m)$, trivial derivation and multiplication given by $p_{i} p_{j}=C_{i+j, i} p_{i+j}$ (where $C_{i+j, i}$ is the binomial coefficient).

If $\Lambda=Z_{p}$ it is an easy exercise with binomial coefficients to show

$$
P(2 n, m) \cong T(2 n, m) \otimes T(2 n p, m p) \otimes \cdots \otimes T\left(2 n p^{j}, m p^{j}\right) \otimes \cdots
$$

where $T(2 k, j)$ is the polynomial algebra on a single generator $\omega$ of bidegree $(2 k, j)$ truncated by the relation $\omega^{p}=0$.

There are also certain combinations of these algebras which we need.

DefinItion 2.3. The special Grassmann algebra $E_{l}(2 n+1, m)$ is isomorphic to $E(2 n+1, m) \otimes P(2(n+1), m)$ as an algebra, but $\partial 1 \otimes p_{j}=$ he $\otimes p_{j-1}$. The integral homology of $E_{h}(2 n+1, m)$ is generated by the elements $\left\{e \otimes p_{j}\right\}$, each generating a cyclic subgroup of order $h$. The homology ring is trivial.

There is one more algebra we will need, the special polynomial algebra $P_{h}(2 n, m)$ (where $h=p^{j} p$ a prime), but it is enormously complex. It has the form

$$
P(2 n, k) \otimes \cdots \otimes E\left(q^{j} 2 n+1, q^{j} k\right) \otimes P\left(q^{j} 2 n+2, q^{j} k\right) \otimes \cdots
$$

where $q$ runs over all primes not equal to $p$, and a very complex derivation which I will not specify. It is best described by specifying its homology ring over $Z$. $H_{*}\left(P_{n}(2 n, k)\right)$ has as generators the elements $\left\{p_{j}\right\}\left(p_{j} \in P(2 n, k)\right)$ with order $\left\{p_{j}\right\}=h v_{j}(p)\left(v_{j}(p)=p^{k}\right.$ where $j=p^{k} m$ with $m$ prime to $\left.p\right)$.

The technique used for computing $H_{*}(B(A))$ for these rings is substantially the same in all cases. Let $M, N, K$ be D.B.A-algebras for which $M \otimes_{\Lambda} N=K$ as a bigraded algebra, the inclusion $i: M \rightarrow M \otimes 1 \subset K$, and the projection $j=$ $\varepsilon \otimes 1: K \rightarrow N$ are both D.B.A-maps. A filtration, and hence a spectral sequence, for $K$ is defined by setting $\mathscr{F}^{i}(K)=j^{-1}\left(N_{0}+N_{1}+\cdots+N_{i}\right)$ (that is, $j^{-1}$ of the $i$-skeleton of $N)$. It is easily verified that $E_{p q}^{1}\left(\mathscr{F}^{i}\right)=H_{q}(M) \otimes N_{p}$ and $E_{p q}^{2}\left(\mathscr{F}^{i}\right)$ $=H_{p}\left(N, H_{q}(M)\right)$. Moreover, in the case at hand the higher boundaries $d_{i}$ may be explicitly given, and from knowledge of the $E^{\infty}$ terms (and the explicit generators) the structure of $H_{*}(N)$ can be inferred.

The $N$ that we use will only be a divided polynomial algebra or a Grassmann algebra (as these are, in a sense, the only algebras which occur in the bar construction). They have the advantage that their homology is free over $\Lambda$, thus the $E^{2}$ terms are relatively simple. The procedure is to use an $N$ to kill the smallest dimensional homology groups in $M$, then iterate the process for $K$, etc. till we have an acyclic complex. It is a remarkable fact that this procedure actually works! 
Applying this technique and assuming $\Lambda=Z$ we obtain the following four lemmas:

LEMMA 2.4. $B(E(2 n-1, m))=P(2 n, m)$.

LEMMA 2.5. There is a construction with initial algebra $P(2 n, m)$ and final algebra $E(2 n+1, m) \otimes E_{2}(4 n+1,2 m) \otimes \cdots \otimes E_{p}\left(2 p^{j} n+1, p^{j} m\right) \otimes \cdots$

where $p$ runs over all primes and $1 \leqq j<\infty$.

LEMMA 2.6. There is a construction with initial algebra $E_{h}(2 n-1, m)$ and final algebra

$$
P_{h}(2 n, m) \otimes E_{p}(2 n p+1, p m) \otimes \cdots \otimes E_{p}\left(2 n p^{j}+1, p^{j} m\right) \otimes \cdots
$$

where $p$ is a prime and $h=p^{k}$.

LEMMA 2.7. With $p, h$ as above there is a construction with initial algebra $P_{h}(2 n, m)$ and final algebra

$$
E_{h}(2 n, m) \otimes E_{p}(2 n p+1, m p) \otimes \cdots \otimes E_{p}\left(2 n p^{j}+1, m p^{j}\right) \otimes \cdots
$$

In the case $\Lambda=Z_{p}$ the results are much simpler, the only algebras which occur are of the form $E(2 n+1, m)$ or $T(2 n, m)$. For these we have

LEMMA 2.8. There is a construction having $E(2 n+1, m)$ as initial algebra and

$$
T(2(n+1), m) \otimes T(2(n+1) p, p m) \otimes \cdots \otimes T\left(2(n+1) p^{j}, p^{j} m\right) \otimes \cdots
$$

as final algebra.

[This is an immediate consequence of 2.4.]

LEMMA 2.9. There is a construction with initial algebra $T(2 n, m)$ and final algebra

$$
E(2 n+1, m) \otimes T(2(n p+1), m p) \otimes \cdots \otimes T\left(p^{j} 2(n p+1), p^{j} m\right) \otimes \cdots
$$

This completes the necessary calculations.

3. Cell decompositions and suspension. Throughout this section we will assume that $X$ is a countable $\mathrm{CW}$-complex with a distinguished 0 -cell $*$.

DEFINITION 3.1. $X$ has a normal filtration if there are subcomplexes $X_{0} \subset X_{1} \subset X_{2}$ $\subset \cdots \subset X_{n} \subset \cdots$ with union $X$ so that $* \in X_{0}$.

If $X$ has a normal filtration put

$$
X_{k, j}=\left(k \text {-skeleton of } X_{j}\right) \cup X_{j-1}
$$

and

$$
C_{k, j}=H_{k}\left(X_{k, j}, X_{k-1, j}\right) \text {. }
$$

The $C_{k, j}$ are all free $\Lambda$-modules and if we set $C_{j}=\sum_{k} C_{k, j}, C_{j}$ becomes a chain complex with boundary operator equal to that in the sequence of the triple $\left(X_{k, j}, X_{k-1, j}, X_{k-2, j}\right)$. 
Proposition 3.2. $H_{k}\left(C_{j}\right)=H_{k}\left(X_{j}, X_{j-1}\right)$.

(This is a special case of Theorem 2.6.10, p. 80 of [9].)

Now suppose that $X$ has a commutative, associative multiplication with unit *

$$
M: X \times X \rightarrow X
$$

$M$ is normal in case: (1) $M$ is cellular, and (2) $M\left(X_{i} \dot{\times} X_{j}\right) \subset X_{i+j}$.

Proposition 3.3. If $X$ has a normal filtration and multiplication then $C=\sum_{k, j} C_{k, j}$ has the structure of a D.B.A-algebra.

Proof. $X_{k-1, j}$ is a neighborhood deformation retract in $X_{k, j}$; hence we may apply the relative Eilenberg-Zilber theorem to

$$
H\left[\left(X_{k, j}, X_{k-1, j}\right) \times\left(X_{m, n}, X_{m-1, n}\right)\right]
$$

and since $C_{k, j}$ is free over $\Lambda$ it follows that there is a natural equivalence

$$
\alpha: C_{k, j} \otimes C_{m, n} \rightarrow H_{k+m}\left[\left(X_{k, j} X_{k-1, j}\right) \times\left(X_{m, n}, X_{m-1, n}\right)\right]
$$

and $\alpha \partial^{\otimes}=\partial \alpha$. We define the ring structure

$$
\mu: C_{k, j} \otimes C_{m, n} \rightarrow C_{k+m, j+n}
$$

by $u=M_{*} \alpha$. Then the naturality of $\alpha$ assures that $u$ makes $C$ into a D.B.A-algebra.

Now, we apply these results to symmetric products. There is an inclusion

$$
S P^{n}(X) \subset S P^{n+1}(X)
$$

obtained by identifying $\left\langle x_{1} \cdots x_{n}\right\rangle$ with $\left\langle x_{1} \cdots x_{n}, *\right\rangle . S P^{\infty}(X)$ is then defined to be the union of the $S P^{n}(X)$ with the weak topology. The monoid structure in the disjoint union of the $S P^{n}(X)$ induces in $S P^{\infty}(X)$ an abelian, associative multiplication with unit $*$. Thus $S P^{\infty}(X)$ is always a filtered space with multiplication.

THEOREM 3.4. Suppose $S P^{\infty}(X)$ has a $\mathrm{CW}$-decomposition with $*$ the only 0-cell so that the filtration and multiplication are normal, then $\operatorname{SP}^{\infty}(\Sigma X)$ inherits these properties and there is an isomorphism of D.B.A-algebras

$$
J: C\left(S P^{\infty}(\Sigma X)\right) \rightarrow B\left(C\left(S P^{\infty}(X)\right)\right) .
$$

[Here $\Sigma X$ is the reduced suspension of $X$.]

Proof. There is an inclusion

$$
j_{n}: \Sigma S P^{n}(X) \rightarrow S P^{n}(\Sigma X)
$$

defined by $j_{n}\left(t\left\langle x_{1} \cdots x_{n}\right\rangle\right)=\left\langle\left(t x_{1}\right), \ldots,\left(t x_{n}\right)\right\rangle$, and this extends to a map $j: \Sigma S P^{\infty}(X) \rightarrow S P^{\infty}(\Sigma X)$. Moreover, there are the maps $j^{(n)}\left(\Sigma S P^{\infty}(X)\right)^{n} \rightarrow S P^{\infty}(\Sigma X)$ where $j^{n}=M(j \times \cdots \times j)$. The images of the successive $j^{(n)}$ contain the preceding ones and form a filtration of $S P^{\infty}(\Sigma X)$ "transverse" to the usual one.

There is a map $F_{n}: I^{n} \times\left(S P^{\infty}(X)\right)^{n} \rightarrow\left(\Sigma S P^{\infty} X\right)^{n}$ which is a relative homeomorphism on interior $I^{n}$,

$$
F\left(t_{1} \cdots t_{n} y_{1} \cdots y_{n}\right)=\left(\left(t_{1} y_{1}\right) \cdots\left(t_{n} y_{n}\right)\right)
$$


Now $I^{n}$ may be triangulated by defining

$$
\sigma_{\alpha}^{n}=\left\{\left(t_{1} \cdots t_{n}\right) \in I^{n}: t_{\alpha^{-1}(1)} \leqq t_{\alpha^{-1}(2)} \leqq \cdots \leqq t_{\alpha}^{\left.-1_{(n)}\right)}\right\}
$$

where $\alpha$ is an element of the symmetric group $\mathscr{S}_{n}$, and $j^{n} F_{n} \mid$ int $\sigma_{\alpha}^{n} \times\left(S P^{\infty}(X)\right)^{n}$ is a relative homeomorphism. Also, $\operatorname{im} j^{n} F_{n}\left|\sigma_{\alpha}^{n} \times\left(S P^{\infty}(X)\right)^{n}=\operatorname{im} j^{n} F_{n}\right| \sigma_{\beta}^{n}$ $\times\left(S P^{\infty}(X)\right)^{n}$. Thus the images im $\left(j^{n} F_{n} \mid \sigma_{1}^{n} \times\left(S P^{\infty}(X)\right)^{n}\right)$ for $n=1,2, \ldots$ decompose $S P^{\infty}(\Sigma X)$.

We would now like to say the images $j^{n} F_{n} \mid \sigma_{1}^{n} \times\left(E^{1} \times \cdots \times E^{n}\right)$ where the $E^{i}$ are cells of $S P^{\infty}(X)$ gives a $C W$-decomposition of $S P^{\infty}(\Sigma X)$. This follows from

LEMMA 3.5. $j^{n} F_{n}=j^{n-1} F_{n-1}\left(D_{j} \times M_{j}\right)$ when restricted to $\sigma_{j(1)}^{n} \times\left(S P^{\infty}(X)\right)^{n}$ where $D_{j}\left(t_{1} \cdots t_{n}\right)=\left(t_{1} \cdots \hat{t}_{j} \cdots t_{n}\right)$

$$
M_{j}\left(y_{1} \cdots y_{n}\right)=\left(y_{1} \cdots M\left(y_{j}, y_{j+1}\right) \cdots y_{n}\right) .
$$

[Here $\sigma_{j(1)}^{n}$ are those points of $\sigma_{1}^{n}$ for which $t_{j}=t_{j+1}$.]

The proof follows from the definitions.

This gives the desired CW-decomposition. It is easy to check that the usual filtration is normal for it, and it remains to check that $M$ is cellular.

LeMmA 3.6. $M\left(j^{n} F_{n} \times j^{r} F_{r}\right)\left|\sigma_{1}^{n} \times\left(S P^{\infty}(X)\right)^{n} \times \sigma_{1}^{r} \times\left(S P^{\infty}(X)\right)^{r} \subset j^{n+r} F_{n+r}\right| \sigma_{1}^{n+r} \times$ $\left(S P^{\infty}(X)\right)^{n+r}$ and is cellular with cellular approximation given by the formula

$$
\begin{aligned}
M_{*}\left\{\sigma^{n} \times E^{1} \times \cdots \times E^{n}\right\} & \otimes\left\{\sigma^{r} \times E^{n+1} \times \cdots \times E^{n+r}\right\} \\
& =(-1)^{r r} \sum_{\alpha}(-1)^{\alpha}\left\{\left(\sigma^{n+r} \times \operatorname{shuff} \alpha E^{1} \times \cdots \times E^{n+r}\right)\right\}
\end{aligned}
$$

where $\tau=\sum_{k=1}^{n} \operatorname{dim} E^{k}$ and $\alpha$ runs over all $(n, r)$ shuffles.

Proof. The first part is clear. For the second consider

$$
\begin{gathered}
\sigma_{1}^{n} \times E^{1} \times \cdots \times E^{n} \times \sigma_{1}^{r} \times E^{n+1} \times \cdots \times E^{n+r} \stackrel{\text { shuff }}{\longrightarrow} \sigma_{1}^{n} \times \sigma_{1}^{r} \times E^{1} \times \cdots \times E^{n+r} \\
M \downarrow \\
S P^{\infty}(\Sigma X) \longleftarrow j^{n+r} F_{n+r} \\
\downarrow
\end{gathered}
$$

In $\sigma_{1}^{n} \times \sigma_{1}^{r}$ a triangulation is induced by the inclusion, and $\sigma_{1}^{n} \times \sigma_{1}^{r}=\sum_{\alpha} \sigma_{\alpha}^{n+r}$ where $\alpha$ runs over all $(n, r)$ shuffles. The proof is now completed by observing that the following diagram is commutative.

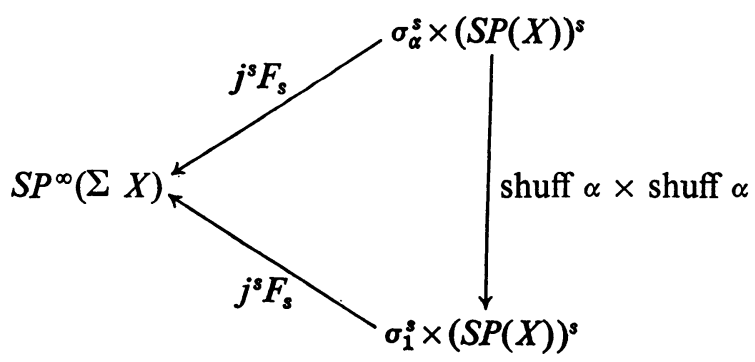


Now, to complete the proof of the theorem set $J\left\{\sigma^{n} \times E^{1} \times \cdots \times E^{n}\right\}=$ $\left[\left\{E^{1}\right\} \otimes \cdots \otimes\left\{E^{n}\right\}\right]$ in $B\left(C\left(S P^{\infty}(X)\right)\right)$. From 3.6 it follows that this is a map of bigraded algebras. The fact that it is a chain map follows from 3.5 and the relative Eilenberg-Zilber theorem.

To complete this section we show that for a great many $X$ (and all those we need) $S P^{\infty}(X)$ admits a CW-decomposition to which the multiplication and filtration are normal.

THEOREM 3.7. Let $C$ be a countable semisimplicial complex with a single generator in dimension 0 , and $|C|$ its geometric realization, then $S P^{\infty}(|C|)$ admits a $\mathrm{CW}$ decomposition satisfying the hypothesis of 3.4 .

[For the definition and properties of $|C|$ see [7].]

Proof. Define $S P^{n}(C)=C \times \cdots \times C / S(n)$, its generators are of the form

$$
\begin{gathered}
\left\langle c_{1} \cdots c_{n}\right\rangle, \quad c_{i} \text { a generator of } C, \\
\partial_{j}\left\langle c_{1} \cdots c_{n}\right\rangle=\left\langle\partial_{j} c_{1} \cdots \partial_{j} c_{n}\right\rangle, \\
s_{k}\left\langle c_{1} \cdots c_{n}\right\rangle=\left\langle s_{k} c_{1} \cdots s_{k} c_{n}\right\rangle .
\end{gathered}
$$

It follows from Theorem 2.2 of [7] that

$$
\left|S P^{n}(C)\right|=S P^{n}(|C|)
$$

and if $* \in|C|$ is the point corresponding to the 0 -dimensional element in $C$, then the decompositions are compatible with inclusion. Finally, it is an easy calculation similar to that in the proof of 3.5 to show the multiplication is normal.

4. The homology rings $\mathscr{R}(X)$ for Moore spaces. $A(\pi, 1)$ is the geometric realization of a countable s.s.c. with a single 0 -dimensional generator, and we may apply the results of the last section.

THEOREM 4.1. (i) There is a D.B.A-homomorphism $E(1,1) \rightarrow C(A(Z, 1))$ inducing isomorphisms in homology.

(ii) There is a D.B.A-homomorphism $E_{h}(1,1) \rightarrow C\left(A\left(Z_{h}, 1\right)\right)$ inducing isomorphisms in homology.

Proof. (i) By the theorem of Steenrod mentioned in the introduction, the injection

$$
j: A(Z, 1) \rightarrow S P^{\infty}(A(Z, 1))
$$

induces an injection in homology onto a direct summand. On the other hand, both these spaces are $K(Z, 1)$ 's. Thus $j_{*}$ is an isomorphism and it suffices to map the nontrivial generator of $E(1,1)$ onto the chain representing a homology generator.

(ii) $S P^{\infty}\left(A\left(Z_{h}, 1\right)\right)$ is a $K\left(Z_{h}, 1\right)$. Its homology groups are known to be $Z$ in dimension 0 and $Z_{h}$ in each odd dimension.

Let $A\left(Z_{h}, 1\right)=|C|$, we may also assume that the dimension of $|C|$ as a $\mathrm{CW}$ complex is 2 . The normalized chain complex $C_{N}$ is the quotient of the chain complex 
of $C$ by the complex generated by degenerate elements. It follows from [7] that $C_{N}$ is isomorphic to the $\mathrm{CW}$-chain complex of $|C|$. In particular $C_{N}^{n}$ has dimension $2 n$ and its top dimensional cells are permuted freely among themselves under the action of $S_{n}$. The singular locus is the set of cells of $C_{N}^{n}$ on which $S_{n}$ does not act freely, it is generated by cells $c_{1} \times \cdots \times c_{n}$ with 2 or more of the $c_{j}$ equal.

Let $\pi: C_{N}^{n} \rightarrow S P^{n}(C)_{N}$ be the projection $\pi\left(c_{1} \times \cdots \times c_{n}\right)=\left\langle c_{1} \cdots c_{n}\right\rangle$. There is a chain map $T: C_{N}^{n} \rightarrow C_{N}^{n}$ defined by $T\left(c_{1} \times \cdots \times c_{n}\right)=\sum_{\alpha} c_{\alpha(1)} \times \cdots \times c_{\alpha(n)}$ where $\alpha$ runs over $S_{n}$. It is clear that $\pi T=n ! \pi$, and defining $\rho\left\langle c_{1} \cdots c_{n}\right\rangle=$ $T\left(c_{1} \times \cdots \times c_{n}\right), \rho$ is a chain isomorphism $S P^{n}(C)_{N} \rightarrow \operatorname{im} T$.

Finally, we need an explicit approximation for the Eilenberg-Zilber theorem,

$$
C_{N} \otimes \cdots \otimes C_{N} \stackrel{P^{(n)}}{\longrightarrow}(C \times \cdots \times C)_{N} .
$$

This is given in case $n=2$ by

$$
P^{2}\left(\sigma^{n} \otimes \sigma^{m}\right)=\Sigma(-1)^{\alpha} s_{\alpha(m+n)} \cdots s_{\alpha(m+1)} \sigma^{n} \times s_{\alpha(m)} \cdots s_{\alpha(1)} \sigma^{m}
$$

where $\alpha$ runs over all $(n, m)$ shuffles. $P^{n}$ is obtained from this by iteration. As a consequence $P^{n} \alpha=\alpha P^{n}$ for any $\alpha$ in $S_{n}$, for details see [5].

Now, let $E$ be a chain in $C_{N}$ representing the one-dimensional generator in $H_{*}(|C|)$, and $F$ is a chain so that $\partial F=h E$. Set $F^{n}=F \otimes \cdots \otimes F$ in $\left(C_{N}\right)^{n}$ and $E^{n}=(1 / h) \partial\left(F^{n}\right)$.

It is easy to see that $P^{n}\left(E^{n}\right)$ and $P^{n}\left(F^{n}\right)$ both belong to im $T$ in $\left(C^{n}\right)_{N}$ and are disjoint from the singular locus. Thus

$$
\pi P^{n}\left(E^{n}\right)=n ! l_{n}, \quad \pi P^{n}\left(F^{n}\right)=n ! f_{n}
$$

and $\rho\left(l_{n}\right)=P^{n}\left(E^{n}\right), \rho\left(f_{n}\right)=P^{n}\left(F^{n}\right)$. On the other hand $E^{n}$, and hence $P^{n}\left(E^{n}\right)$ represents a nontrivial element of order $h$ in $H_{*}\left(|C|^{n}\right)$, thus the same is true of $l_{n}$ for $H_{2 n-1}\left(S P^{n}|C|\right)$.

Thus $l_{n}$ also represents a nontrivial element of order $h$ in

$$
H_{2 n-1}\left(S P^{n}(|C|), S P^{n-1}|C|\right)
$$

and it follows that for $j \neq 2 n-1, H_{j}\left(S P^{n}(|C|), S P^{n-1}(|C|)\right)=0$.

Finally, we have

$$
(n+m) ! f_{n+m}=\pi P^{n+m}\left(F^{n} \otimes F^{n}\right)=M P^{n} \otimes P^{m}\left(F^{n} \otimes F^{m}\right)=n ! m ! f_{n} \cdot f_{m}
$$

since $P$ is natural. Thus $f_{n} \cdot f_{m}=\left(C_{n+m, n}\right) f_{n+m}$. Also,

$$
n ! l_{n}=\pi P^{n}\left(E^{n}\right)=n \pi P^{n}\left(E \otimes F^{n-1}\right)=n\left(M l_{1} \otimes(n-1) ! f_{n-1}\right)=n ! l_{1} f_{n-1},
$$

and the proof is complete.

To handle the case of $\mathscr{R}(A(\pi, n)) n>1$ observe that $A(\pi, n)=\Sigma(A(\pi, n-1))$. Theorem 3.4 now may be applied, and the study of the resultant D.B.A-algebra is easily accomplished with the techniques and results of the first two sections. 
To give the final results we need some notation. Let $T_{k}(n)$ be the set of ordered sequences of positive integers $\left(t_{1}, \ldots, t_{k}\right)$ with $\sum_{j=1}^{k} t_{j}=n$ (ordered partitions of $n$ ). Let $Z^{+}$be the positive integers, and $C_{k}$ the $k$-fold Cartesian product of $Z^{+}$with itself. We now define functions $A_{p}, B_{p}$ from $T_{k}(n) \times C_{k-1}$ to $Z^{+}$as follows:

$$
\begin{aligned}
A_{p}\left[\left(t_{1}, \ldots, t_{k}\right)\right. & ,(s(1), \ldots, s(k-1))] \\
& =t_{k}+p^{s(k-1)}\left\{\varepsilon_{k-1}+t_{k-1}+p^{s(k-2)}\left[\varepsilon_{k-2}+t_{k-2}+\cdots+p^{s(1)}\left(t_{1}+\varepsilon_{1}\right) \cdots\right]\right\}
\end{aligned}
$$

(here $\varepsilon_{i}=0$ if $t_{i}$ is even and $\varepsilon_{i}=1$ if $t_{i}$ is odd). $B_{p}\left[\left(t_{1}, \ldots, t_{k}\right),(s(1), \ldots, s(k-1))\right]$ $=p^{s(1)+\cdots+s(k-1)}\{(A(x, y), B(x, y))$ will be the bidegree of the generator associated to $(x, y)\}$.

Now we must isolate three further properties of the pairs in $T_{k}(n) \times C_{k-1}-(x, y)$ is of type 1 if $x=\left(t_{1}, \ldots, t_{k}\right)$ and $t_{k}$ is odd, it is of type 2 if $t_{k}$ is even, and of type 3 if $t_{1}$ is even.

Notice that $A_{p}(x, y)$ is odd only if $(x, y)$ is of type 1 .

Now, for each prime $p$ set $J_{n}(p)$ equal to the tensor product of the rings $P_{p}\left(A_{p}(x, y), B_{p}(x, y)\right)\left[E_{p}\left(A_{p}(x, y), B_{p}(x, y)\right)\right]$ as $(x, y)$ runs over $\bigcup_{k=2}^{n} T_{k}(n) \times C_{k-1}$ and is of type 2 [respectively of type 1].

Similarly $K_{n}(p)$ is the tensor product of rings $P_{p}\left(A_{p}(x, y), B_{p}(x, y)\right)$ [respectively $\left.E_{p}\left(A_{p}(x, y), B_{p}(x, y)\right)\right]$ as $(x, y)$ runs over all elements in $\bigcup_{k=2}^{n} T_{k}(n) \times C_{k-1}$ and is simultaneously of types 2 and 3 [respectively of types 1 and 3].

Now we have

THEOREM 4.2. (i) For $n$ even

$$
\begin{aligned}
& \mathscr{R}(A(Z, n) ; \Gamma)=H_{*}\left(P(n, 1) \otimes \bigotimes_{p \text { prime }} K_{n}(p) ; \Gamma\right), \\
& \mathscr{R}\left(A\left(Z_{h}, n\right) ; \Gamma\right)=H_{*}\left(P_{h}(n, 1) \otimes J_{n}(p) ; \Gamma\right),
\end{aligned}
$$

where $h=p^{j}$ ( $p$ prime).

(ii) For $n$ odd

$$
\begin{aligned}
\mathscr{R}(A(Z, n) ; \Gamma) & =H_{*}\left(E(n, 1) \otimes{\underset{p}{\text { prime }}}_{\otimes_{n}} K_{n}(p) ; \Gamma\right), \\
\mathscr{R}\left(A\left(Z_{h}, n\right) ; \Gamma\right) & =H_{*}\left(E_{h}(n, 1) \otimes J_{n}(p) ; \Gamma\right),
\end{aligned}
$$

and if $\Gamma$ is $Z$ or $Z_{q}$ with $q$ prime this is an isomorphism of bigraded rings.

(The proof is a direct induction using 4.1 to start and the calculations of $\S 2$ to continue.)

For the special case where $\Gamma=Z_{p}$ the reader will have little difficulty specifying the resultant algebra as the tensor product of $T(r, s)$ 's and $E(q, t)$ 's where $(r, s)$, $(q, t)$ run over a somewhat larger set than the corresponding index set in the integer case.

Moreover, one could easily use the fact that the Steenrod algebra $A(p)$ is transgressive in the Serre spectral sequence to obtain new proofs of some results of Nakaoka on the action of the Steenrod algebra in $S P^{n}(M)$ where $M$ is one of the other Moore spaces considered here. 
ONE FURTHER REMARK. The use of the Thom-Dold theorem in the proof of 4.1 was not essential. It is possible in fact with a little more work to use the results of Cartan [2] and 4.2 to show $S P^{\infty}\left(A\left(Z_{h}, n\right)\right)$ is a $K\left(Z_{h}, n\right)$ directly, thus obtaining an independent proof of part of the Thom-Dold theorem.

5. The homology of $S P^{n}(X)$. Dold has shown [4] that $H_{*}\left(S P^{n}(X)\right)$ depends only on $H_{*}(X)$. On the other hand, given an arbitrary arcwise connected space $X$ of finite type, there is a wedge product $Y$ of Moore spaces with isomorphic homology groups. Thus $H_{*}\left(S P^{n}(X)\right)=H_{*}\left(S P^{n}(Y)\right)$ and the problem reduces to calculating $\mathscr{R}(Y ; \Gamma)$ where $Y$ is a wedge product.

TheOREM 5.1. Let A, B be topological spaces which satisfy the hypothesis of 3.4, then

$$
\mathscr{R}(A \vee B) \cong H_{*}(C(A) \otimes C(B))
$$

the isomorphism being of bigraded algebras.

Proof. $A, B$ are $\mathrm{CW}$-complexes, thus they have contractible neighborhoods of their respective base points.

The mappings $\rho_{1}: A \rightarrow A \vee B, \rho_{2}: B \rightarrow A \vee B$ induce a mapping $\rho: S P^{\infty}(A)$ $\times S P^{\infty}(B) \rightarrow S P^{\infty}(A \vee B)$. Let $T_{n}=\rho^{-1} S P^{n}(A \vee B)$, then $T_{n-1}$ is a neighborhood deformation retract in $T_{n}$ as is $S P^{n-1}(A \vee B)$ in $S P^{n}(A \vee B)$. Thus we can take excisions, and

$$
\begin{aligned}
T_{h}-T_{h-1} & \cong S P^{n}(A \vee B)-S P^{n-1}(A \vee B) \\
& =\sum_{j=0}^{n}\left(S P^{j}(A)-S P^{j-1}(A)\right) \times\left(S P^{n-j}(B)-S P^{n-j-1}(B)\right) .
\end{aligned}
$$

The result now follows from the relative Eilenberg-Zilber theorem and the fact that $\rho$ commutes with multiplication.

COROLlaRY 5.2. Let $\pi$ be a finitely generated abelian group then $\mathscr{R}(A(\pi, n))$ is isomorphic to $H_{*}(G(\pi, n))$ where $G(\pi, n)$ is an explicitly given tensor product of the D.B.A-algebras of 4.2 .

Proof. $A(\pi, n)$ may be represented as a wedge product of $A\left(Z_{h}, n\right)$ 's and $A(Z, n)$ 's. As an application of these results let $X$ be a Riemann surface of genus $g$. Then

$$
\mathscr{R}(X) \cong E(11) \otimes \cdots \otimes E(11) \otimes P(21) \quad \text { (where } E(11) \text { appears } 2 g \text { times). }
$$

Thus $\mathscr{R}(X)$ has no torsion, and the same is true for $S P^{n}(X)$. The Betti number of $H_{k}\left(S P^{n}(X)\right)$ is equal to the number of ways we can have

$$
\varepsilon_{1}+\cdots+\varepsilon_{2 g}+r \leqq n
$$

subject to the condition

$$
\varepsilon_{1}+\cdots+\varepsilon_{2 g}+2 r=k
$$


where $\varepsilon_{i}=0$ or 1 . This is easily seen to be

$$
\sum_{r=0}^{[k / 2]}\left(\begin{array}{c}
2 g \\
k-2 r
\end{array}\right) .
$$

\section{BIBLIOGRAPHY}

1. H. Cartan, Sur les groupes d'Eilenberg-MacLane $H(\pi, n)$. I, Proc. Nat. Acad. Sci. U.S.A. 40 (1954), 467-471.

2. - Sur les groupes d'Eilenberg-MacLane. II, Proc. Nat. Acad. Sci. U.S.A. 40 (1954), 704-707.

3. - Séminaire H. Cartan (Exposé 3), Secrétariat Math., Paris, 1954-1955.

4. A. Dold, Homology of symmetric products and other functors of complexes, Ann. of Math. 68 (1958), 54-80.

5. - Decomposition theorems for S(n)-complexes, Ann. of Math. 75 (1962), 8-16.

6. S. Eilenberg and S. MacLane, On the groups $H(\pi, n)$. I, Ann. of Math. 58 (1953), 58-106.

7. J. Milnor, The geometric realization of a semi-simplicial complex, Ann. of Math. 65 (1957), 357-362.

8. R. Thom and A. Dold, Quasifaserungen und unendliche symmetrische Produkte, Ann. of Math. 67 (1958), 239-281.

9. G. W. Whitehead, Homotopy theory, Mimeographed Notes, Massachusetts Institute of Technology, Cambridge, 1955.

10. R. J. Milgram, The bar construction and Abelian H-spaces, Illinois J. Math. 11 (1967), 242-250.

University of Illinors at Chicago Circle,

Chicago, Illinois

Princeton University,

Princeton, New Jersey 